\title{
CORRESPONDENCE
}

\section{ACCOMMODATION IN FISHES}

To the Editors of The British Journal of Ophthalmology.

SIRS, - I am much obliged to Mr. M. S. Mayou for his courtesy and for the trouble he has taken to state explicitly the facts he has elicited with regard to fresh-water fish.

Professor Osborne has drawn my attention to the fact that Hess noted failure to produce any change on electrical stimulation of a sturgeon's eye.

It is obvious that a complete survey of the refraction and accommodation (where it exists) in the eyes of all fishes is desirable and should be made with the fish in water. This might be done at a Marine Biological Station where the necessary facilities are available.

The variation in the accommodation and necessary structures from Cephalapods to Primates is so remarkable in the apparently causeless variations that nothing new would surprise any one who had looked into the problem. The existing knowledge raises the fundamental biological problem. Why should these suppressions and variations occur and what is the cause? If and when an answer can be given it will illuminate many other biological conditions at present unexplainable.

It is my misfortune that a geographical factor, viz., a distance of 13,000 miles renders a full personal discussion of this fascinating problem impossible.

JAMES W. BARRETt.

MELBourne,

May, 1933.

To the Editors of The British Journal of Ophthalmology.

DEAR SIRS,-I shall be obliged if you will insert a few lines about my new instruments in your journal.

A new forceps for intracapsular extraction of cataract.-The forceps is made of stainless steel and is given a double curve at the end, so that without undue depression it is easily brought to bear on the anterior surface of the lens.

At each end there is a bisected cup and on closure of the blades there is perfect apposition of the cups. The tips are blunt so that there is no danger of injuring the capsule. 


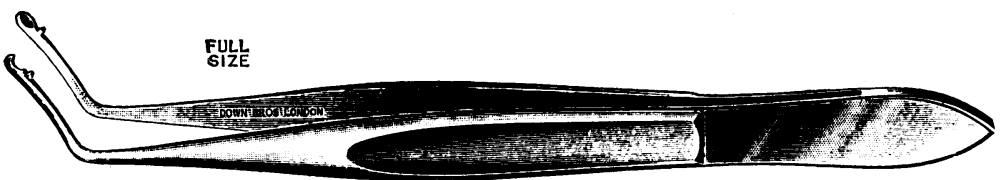

When the capsule is caught between the cups it forms a knuckle which can be easily seen through the cornea. Once the capsule is gripped it seldom slips or tears provided the cataract is of a suitable variety for intracapsular extraction.

I have done 150 extractions with this forceps with splendid results.

This forceps is made by Messrs. Down Bros., of London, and is a very handy and well finished instrument.

The hook or lens expressor.-This instrument is like an ordinary tenotomy hook with a knob at the end; but the curve is so modified

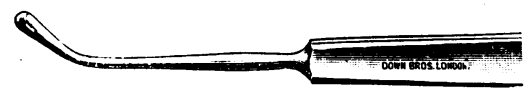

that gentle pressure can be easily applied in a desired direction without interference from the surrounding parts. This expressor is also made by Messrs. Down Bros.

Whilst reading the Archives of Ophthalmology of November, 1932, I came across the description of a special scissors by B. Treutler. The same idea had struck me at about the same time in 1931, and I had asked Messrs. Down Bros., of London, to make a cystotome scissors for me. The main idea was to make the outer edges and the point of De Wecker's scissors sharp, so that with closed blades you can pierce the cornea and capsule. Once you have pierced the capsule it is an easy matter to rip it open by opening the blades and you can regulate the size of the opening in the capsule by your

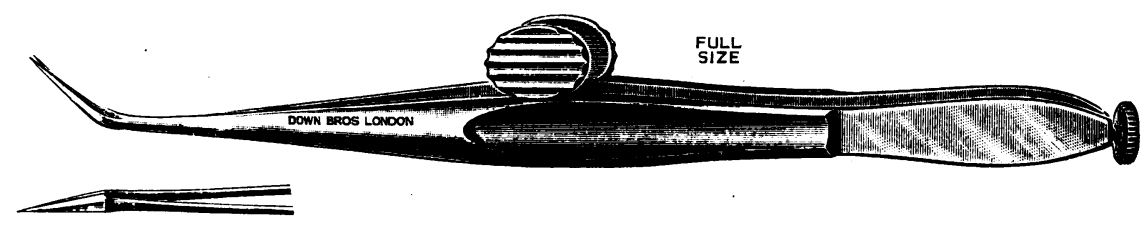

fingers. In this way tough capsules which have resisted the sharpest knives can be easily cut and the force applied being centrifugal there is no drag on the ciliary body and therefore no danger of subsequent 
inflammation. The puncture in the cornea is also very small and heals up rapidly.

I have found this cystotome scissors very useful for iridotomies also.

\section{Yours faithfully,}

R. P. RATNAKAR.

\section{NOTES}

Mr. H. B. Stallard has been elected Assis-

Appointments tant Surgeon to the Royal London Ophthalmic Hospital; and Mr. C. D. Shapland has been elected Assistant Ophthalmic Surgeon to University College Hospital.

\section{FUTURE ARRANGEMENTS}

\section{3}

October 6.-Midland Ophthalmological Society, Annual Meeting, at Birmingham and Midland Eye Hospital.

December 5.-Midland Ophthalmological Society, at Birmingham and Midland Eye Hospital.

\section{CONTEMPORARY OPHTHALMIC LITERATURE}

American Journal of Ophthalmology. June, 1933.

PETER.' The treatment of non-paralytic squint.

FERREE and RAND. The eye as a factor in the difference in hue between daylight and twilight.

Rowland. Visual field studies.

BuRTON ChANCE. Dr. Randall as an ophthalmologist.

PASCAL. Simultaneous comparison in subjective testing.

POE. Ophthalmoplegia totalis.

O'CONNOR. Lens removal for high myopia.

MARLOW. Recent observations on the prolonged occlusion test. 